\title{
Huertas Caseras Familiares: Estrategia para el fortalecimiento de las relaciones interpersonales y la convivencia ${ }^{1}$

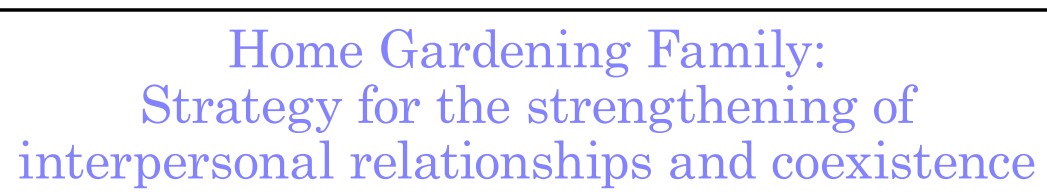

DOI: http://dx.doi.org/10.17981/cultedusoc.9.3.2018.30

Artículo de investigación. Fecha de recepción: 15/06/2018. Fecha de aceptación: 27/11/2018

Alonso Oviedo Garcia; Amira Torres Aragon; Gloria Torres Teran; Magda Ortiz Jimenez; Rosiris Rodriguez Martinez; Marellys Arias Pasos; Marelbys Castillo Hernandez; Maria Buelvas Ospino; Maria Jimenez Villalba; Martha Diaz Mercado; Mary Maldonado Sierra y Minelba Jimenez Ospino² IED María Auxiliadora

Para citar este artículo:

Oviedo, A., Torres, A., Torres, G., Ortiz, M., Rodriguez, R., Arias, M., Castillo, M., Buelvas, M., Jimenez, M., Diaz, M., Maldonado, M. y Jimenez, M. (2018). Huertas Caseras Familiares: Estrategia para el fortalecimiento de las relaciones interpersonales y la convivencia. Cultura. Educación y Sociedad 9(3), 263-272. DOI: http://dx.doi.org/10.17981/cultedusoc.9.3.2018.30

\section{Resumen}

Las huertas caseras familiares son una apuesta colectiva de cuidado del entorno y se convierte en una estrategia saludable de consumo, propiciando la participación de las comunidades educativas. La presente investigación tiene como objetivo identificar los beneficios que genera la creación de huertas caseras familiares, para mejorar las relaciones interpersonales y de convivencia, de la Institución Educativa Departamental Liceo Ariguaní: Sede El Retiro. El estudio se aborda desde una mirada cualitativa, con un alcance descriptivo y con una población constituida por estudiantes, docentes y padres de familia. Se utilizó como técnica la entrevista y la observación. Dentro de las conclusiones se puede destacar que las huertas caseras familiares resultan una estrategia motivadora no solo para los estudiantes, sino para la comunidad educativa en general, siendo un medio de generación de ingresos, un aporte al cuidado del entorno y una contribución al consumo de alimentos naturales y saludables.

Palabras clave: Huertas caseras familiares; relaciones interpersonales; convivencia; cultivos.

\section{Abstract}

The family home gardens are a collective commitment to take care of the environment and become a healthy strategy of consumption, encouraging the participation of educational communities. The present investigation has like objective Identify the benefits that generates to creation of familiar homemade gardens, to improve the interpersonal relations and of coexistence, of the Educational Institution Departmental Liceo Ariguaní: Sede El Retiro. The study is approached from a qualitative perspective, with a descriptive scope and with a population made up of students, teachers and parents. The survey and observation were used as a technique. Among the conclusions we can highlight that family home gardens are a motivating strategy not only for students, but for the educational community in general, being a means of income generation, a contribution to caring for the environment and a contribution to the consumption of natural and healthy foods.

Keywords: Family home gardens relationships; coexistence; crops.

1Este artículo ha sido derivado del Programa de Fortalecimiento de la Cultura Ciudadana y Democrática CT+I a través de la IEP apoyada en TIC en el Departamento de Magdalena: CICLON

2 Docentes miembros del grupo de investigación "Soñadores de la tierra colora” de la IED María Auxiliadora.

- The author; licensee Universidad de la Costa - CUC.

Cultura, Educación y Sociedad vol. 9 no. 3, pp. 263-272. Diciembre, 2018

Barranquilla. ISSN 2389-7724 Online 


\section{Introducción}

En el que hacer diario del proceso de enseñanza-aprendizaje son muchas las experiencias que marcan al a comunidad docente, algunas alegres, que traen consigo grandes satisfacciones, sobre todo cuando logran superar sus propias limitaciones y avanzar en la consecución de sus objetivos. Otras tristes, cuando a pesar de su empeño y el del docente su avanzar es poco o lento y hasta frustrantes. Circunstancias que exigen evaluar los que lo que se hace, como se hace, para que se hace y que resultados se obtienen.

Una autoevaluación hecha a conciencia, permite: primero, entender que está pasando, qué es aquello que nos impide guiar a los estudiantes hasta la meta. Segundo, examinar, proponer, evaluar y seleccionar una o varias alternativas pedagógicas y didácticas que propicien en los estudiantes una nueva fuerza motivadora por el conocimiento y su construcción. Y, tercero, de la mano con esta alternativa redefinir el quehacer y enfocarnos de nuevo. Es así, como empezamos a explorar acerca de una estrategia pedagógica que nos permitiera involucrar varios aspectos fundamentales: investigación, Tics, prenderhaciendo, convivencia y construcción de tejido humano entre los miembros de la comunidad educativa de la Sede El Retiro.

Entre el abanico de posibilidades, fluyeron muchas ideas, referentes de experiencias pasadas, Una vez analizada la problemática de los educandos de la sede educativa El Retiro, se encontraron resultados alarmantes en cuanto al desempeño académico, esto sumado a una gran problemática socio afectiva y económica.
Pensando en una solución que responda a las necesidades de la IED se busca promover e implementar las huertas caseras como estrategia didáctica $e$ investigativa para la enseñanza de una adecuada alimentación en los niños y niñas y toda la comunidad educativa y para que además, ellos se conviertan en actores del proceso, participando activamente en la siembra, sostenimiento, recolección de los frutos y a largo plazo, se apunta a la comercialización de los productos, como una alternativa para generar ingresos a las familias vulnerables de la comunidad.

El propósito de esta investigación es identificar los beneficios que genera la creación de huertas caseras familiares, para mejorar las relaciones interpersonales y de convivencia en la sede educativa, partiendo de los intereses colectivos de la comunidad, pues tal como lo expresa Herrera (2014),

"El interés colectivo se configura, como un interés que pertenece a todos y cada uno de los miembros de una colectividad determinada, el cual se concreta a través de su participación activa ante la administración de justicia, en demanda de su protección de los intereses grupales o masivos"(p. 4).

Es importante valerse de la orientación de expertos que integren a las familias, valoren sus aportes y les capaciten sobre la manera de llevar a cabo las huertas, de las cuales se espera la obtención de: hortalizas, legumbres, frutas, vegetales, que ayuden a fortalecer la sana nutrición en los niños y niñas de la sede y que a su vez esto se refleje en mejores niveles de desempeño académico en nuestros niños y niñas.

Los educandos a través de la implementación de las huertas caseras, participaran de manera activa en la ejecución del proyecto, logrando así ad- 
quirir competencias que les permitan ser capaces de transformar su realidad y de buscar solución a las problemáticas que se presenten en su entorno. Según estudio realizado por la Universidad de Pamplona en el año 2012, las huertas caseras desde hace muchos años atrás ha sido de mucho provecho para sustituir aquellas necesidades alimentarias tanto para su venta y recaudo, logrando asi obtener ganancias para la escuela, hoy en dia es de suma importancia inculcar al estudiante lo significativo que es emprender y fortalecer la producción de huertas.

Esto marca un horizonte en el tiempo e invita a reflexionar sobre el aprovechamiento de las huertas caseras familiares en las sedes educativas donde además de contribuir al mejoramiento de las relaciones interpersonales, la integración de las familias a los procesos formativos de los educandos, el fortalecimiento de la convivencia, el mejoramiento en el desempeño escolar de los niños y la promoción de una sana alimentación para los mismos; también podrían obtener ingresos económicos con la comercialización de productos derivados de las huertas, fomentando el emprendimiento y ayudando a estas familias, que en su mayoría son vulnerables a obtener recursos para su sostenimiento.

No cabe duda que el principal problema que atraviesa la Educación Básica en América Latina es la falta de consentimiento social que acepte que de verdad existe un problema de igualdad y calidad en el proceso de formación.

El Ministerio de Educación Nacional de acuerdo a su política educativa propone acciones que propenden el mejoramiento de la calidad educativa. Cabe mencionar que el artículo 85 de la Ley 115 de 1994 (Ministerio De Educación
Nacional, 1994), conforme se encuentra modificado por el artículo 57 de la Ley 1753 de 2015 (Instituto Agustín Codazzi, 2015) establece que: "El servicio público educativo se prestará en las instituciones educativas en jornada única”. En este sentido el mismo decreto define que la jornada única comprende el tiempo diario que dedica el establecimiento educativo para el desarrollo de las áreas obligatorias y fundamentales, y de las asignaturas optativas, así como el tiempo destinado al almuerzo de los estudiantes

Desde esta perspectiva, se puede evidenciar que la Institución Educativa Departamental Liceo Ariguaní no es ajena a esta situación y en tal sentido El Ministerio de Educación Nacional decidió desde el mes de Julio de 2016 incluir en jornada única a la sede El Retiro de la mencionada institución. Contrario a lo que se pensaría sería la solución a los problemas de tipo socio económico que afectan la convivencia escolar y por ende el desarrollo académico de los estudiantes que asisten a llevar a cabo el proceso de desarrollo del aprendizaje desde transición hasta quinto grado; los problemas aún subsisten y es preocupación tanto de los directivos docentes como de los docentes que a diario laboran en este centro educativo.

Contextualizando la dificultad identificada en la sede el Retiro, se encuentra que la desescolarización de padres de familia y comunidad en general, no ayuda a los niños a crecer en un buen ambiente académico, ya que ellos no consideran el estudio como una opción para el crecimiento de su grupo social o una mejor oportunidad para crecer en el ámbito laboral a futuro. Los estudiantes se encuentran rodeados de situaciones en donde se promueve recurrentemente el conseguir pareja a 
temprana edad, dinero fácil, consumo de sustancias alcohólicas y alucinógenas, embarazos a temprana edad, abuso sexual, entre otros.

Por otro lado también estas situaciones generan como lo manifiesta el autor Cruz (2016) que los indicadores convencionales, como la tasa de homicidios, los enfrentamientos, o incluso el dominio sobre territorios de determinados actores armados no dan cuenta del conflicto y de las variables que involucra en toda su complejidad y, aún si así fuera, pueden tener repercusiones distintas sobre la protesta, facilitarla, provocarla o inhibirla (p. 40)

Buscando dar respuesta entonces a los factores que intervienen en las distintas necesidades de los estudiantes, y a fin de enmarcar la necesidad de la creación de huertas caseras familiares se explica entonces que desde el momento de la gestación, con la alimentación de la madre comienza el efecto de la nutrición en el desarrollo cerebral del niño, lo mismo sucede en la lactancia, donde la madre debe alimentarse muy bien para pasarle nutrientes, vitaminas al lactante. La nutrición es crucial en los primeros años de vida. Puede afirmarse entonces que el desarrollo cognitivo empieza desde la concepción y se extiende a lo largo de toda la vida; donde la alimentación juega un papel muy importante.

La necesidad entonces de investigar se soporta en lo que la cumbre mundial expuso sobre la alimentación en noviembre de 2012 reafirmando el derecho de toda persona a tener acceso a alimentos sanos y nutritivos. Varios países han desarrollado e implementado en reformas constitucionales, leyes nacionales Colombia en su Constitución Política de 1991 reconoce de manera explícita y directa el derecho a la alimentación, siendo éste un Derecho reconocido y aplicable. Es un derecho fundamental de los niños la alimentación equilibrada (Artículo 44) (Constitución Política de Colombia, 1991).

Con base en estos estudios se puede inferir que la alimentación de los niños es una prioridad y debe serlo en las Instituciones Educativas, que es el espacio donde los niños y niñas pasan gran parte de su tiempo y donde además ejercitan su cerebro y su cuerpo en diferentes actividades físicas y cognitivas, las cuales demandan que el educando este bien alimentado y dispuesto a participar en estos procesos.

De acuerdo con Bastidas (2012) referido por Palacios, (2016) la huerta escolar como táctica dinámica tiene como finalidad de motivar e incentivar el espiruto de emprender y la construcción de una enseñanza activa y lograr impulsar en un entorno vivo y físico, donde se aprenderá entre otras cosas a fortalecer la producción nutricional y los beneficios del desarrollo endógeno.

El pensamiento de Bastidas (2012), describe la huerta escolar como ese espacio de interacción entre la construcción del conocimiento científico - empírico y de los procesos nutricionales de los niños y niñas. Es un espacio donde tanto los niños como los padres ocuparan su tiempo libre en actividades productivas que generen beneficios a las familias. Los jóvenes, por ende, se mantendrán alejados de los flagelos sociales circundantes en el entorno de la sede educativa.

Mazzini (2012), referido por Palacios (2016) afirma que la enseñanza es un proceso difícil, que involucra quien enseña y grupo que aprende del contenido, La huerta escolar, es una estrategia de enseñanza en la cual facilita la integración de los contenidos a enseñar, 
logrando que los alumnos aprendan a descubrir, resolver problemas, reflexionar, ayudarse mutuamente buscando construir una propuesta pedagógica coherente que integre tanto a los educandos como a los docentes, comunidad educativa en general.

Es muy relevante el postulado de Mazzini (2012), quien nos deja ver que la huerta escolar además de ser una estrategia para construir aprendizajes significativos, es en sí misma un cumulo de aprendizajes de diferentes áreas del conocimiento que complementan el proceso de enseñanza aprendizaje y que se constituyan como espacios ejecutores de acciones que mejoran la escuela y su entorno, que mejoran además la situación nutricional de los niños y niñas de la sede, como una prioridad partiendo de la realidad de las familias. El conocer las necesidades de los estudiantes, vivir día a día los problemas y flagelos de la comunidad en general invita a potenciar y transformar las necesidades y realidades de la comunidad en general (Vera, 2015).

Según Galvis (2012) referido por $\mathrm{Pa}$ lacios, (2016) durante el proceso de enseñanza aprendizaje, se pretende que los conceptos vistos en el aula, sean llevados a la práctica durante el trabajo en la huerta. y a su vez involucra a los padres de familia para mejorar los hábitos de vida saludables en la comunidad.

Galvis (2012) describe la aplicabilidad de los conocimientos adquiridos en el aula en la implementación de la huerta escolar, pero que aún más allá estos conocimientos se reflejen en un cambio en los hábitos de vida de la comunidad en general, desde la nutrición; procurando que toda la comunidad educativa aprenda y esté al tanto de hábitos saludables de alimentación a través de las huertas escolares. Apoyados en este pensamiento, es importante diseñar estrategias, donde existe la relación familia- escuela, se logren directrices que contribuyen al desarrollo de hábitos saludables y actitudes positivas en favor del desarrollo individual de los niños y niñas durante su etapa escolar.

Las Huertas Caseras es un espacio más bien reducido, dedicados a producir gran cantidad de vegetales útiles como son hortalizas, verduras, legumbres, algunos frutos, especias y medicinales para uso y consumo doméstico. (Gómez y Ortega, 2012) referido por (Gómez, 2015). Estos pequeños espacios que describe se convierten en la escuela en zonas abiertas de interacción entre los educandos y el proceso mismo de enseñanza aprendizaje, donde los niños y niñas a través del cultivo, mantenimiento y cuidado de la huerta, se forman integralmente en conocimientos multidisciplinares, valores y sana convivencia.

La escuela es, de este modo, el principal escenario para satisfacer las necesidades de la salud en el periodo de formación física, psíquica y social de los niños y niñas de la sede a fin de que desarrollen con gran capacidad para el aprendizaje y la asimilación de hábitos saludables.

También es importante que el docente desarrolle habilidades para la implementación de actividades pedagógicas que permita a los estudiantes interactuar directamente con su entorno, aplicando la interdisciplinariedad como objeto principal en los procesos del saber y el saber hacer (Avendaño, Cortés y Guerrero 2015).

Como es de notarse las tecnologías hacen parte de la vida de los individuos, por lo que resulta de gran importancia y como llamado de atención que los estudiantes sean direccionados desde las 
escuelas para que el uso que le den a las herramientas tecnológicas sean desde una mirada pedagógica, es decir con fines educativos que le permitan al interior del aula facilitar la apropiación. Por lo tanto, el presente proyecto de la Huerta escolar se convierte en una alternativa, desde el reconocimiento del medio ambiente y todo lo que nos ofrece, para poder generar nuevos saberes que se articulen a las áreas temáticas que desarrollan los maestros, permitiendo además apoyarse de las tecnologías, y contribuyendo al fortalecimiento de valores, tales como el trabajo en equipo, cooperación, colaboración, altruismo entre otros.

\section{Metodología}

El trabajo de investigación tiene un enfoque cualitativo el cual permite entender y analizar la realidad social conjugando la perspectiva que tienen los sujetos sobre su entorno, el alcance de la investigación es descriptivo.

\section{Escenarios y actores}

El estudio lo conforman la comunidad educativa de la institución Liceo Ariguaní, sede el Retiro. En la participación de la encuesta se contó con 50 miembros distribuidos en 30 estudiantes, 10 padres de familia y 10 docentes.

\section{Técnicas e instrumentos de} recolección de la información

Las técnicas utilizadas el proyecto fueron la Entrevista estructurada y el diario de campo, que permitieron hacer el registro de lo que se observó y detalló en la información suministrada por los participantes.

\section{Procedimiento}

Se construyeron los contenidos de acuerdo de acuerdo a la problemática estudiada. Luego fue enviada a pares expertos para su respectiva validación. Una vez validados se diseñó un instrumento para ser sometido a una prueba piloto, tomando los aportes más significativos de los aspectos evaluados.

Culminada la prueba maestral se envió de nuevo a los jueces expertos, quienes hicieron las correcciones y recomendaciones pertinentes. Finalmente se procede a realizar la aplicación a la población objeto de estudio. El diseño de la Entrevista estructurada se formuló de tal manera que se obtuviera información precisa sobre el objeto de investigación, para identificar los beneficios que genera la creación de huertas caseras familiares, para mejorar las relaciones interpersonales y de convivencia

El diseño de las preguntas, tuvo una guía que permitiera elaboración definitiva del cuestionario y del plan operativo de la entrevista basándose en propósitos específicos de la investigación, se realizó una validación presentada a expertos y fue aplicada a la comunidad educativa de la sede el retiro.

\section{Resultados}

A continuación, se presentan los resultados del estudio lo cual se tuvo en cuenta la pregunta orientadora ¿De qué manera las huertas caseras familiares mejoran la motivación de los educandos frente el proceso educativo y fortalece las relacione interpersonales y de convivencia en la IED Liceo Ari guaní Seccional El Retiro? 


\begin{tabular}{|c|c|c|c|}
\hline $\begin{array}{c}\text { Categoría de } \\
\text { estudio }\end{array}$ & $\begin{array}{c}\text { Pregunta } \\
\text { orientadora }\end{array}$ & Discurso de los actores & Articulación y sistematización teórica \\
\hline Convivencia & $\begin{array}{l}\text { ¿Cuáles } \\
\text { considera son } \\
\text { los factores que } \\
\text { afectan la sana } \\
\text { convivencia de } \\
\text { los integrantes } \\
\text { de la comunidad } \\
\text { educativa } \\
\text { (Escuela- } \\
\text { familia- } \\
\text { Comunidad) de } \\
\text { El Retiro? }\end{array}$ & $\begin{array}{l}\text { Las familias afirmaron que } \\
\text { algunos de los factores que } \\
\text { afectan la sana convivencia } \\
\text { en los integrantes de la } \\
\text { comunidad educativa se } \\
\text { encontraron: la existencia } \\
\text { de hogares disfuncionales, } \\
\text { la precaria situación } \\
\text { económica, la falta de } \\
\text { empleo, alimentación } \\
\text { (necesidades básicas), la } \\
\text { ubicación de la comunidad } \\
\text { educativa alrededor de } \\
\text { la zona de tolerancia, la } \\
\text { discriminación, el irrespeto, } \\
\text { la falta de responsabilidad } \\
\text { de los padres de familia, } \\
\text { el alcoholismo, maltrato } \\
\text { intrafamiliar, la no } \\
\text { utilización del tiempo libre } \\
\text { para fomentar actividades }\end{array}$ & $\begin{array}{l}\text { De acuerdo a los hallazgos encontrados en } \\
\text { la entrevista aplicada, se deduce de manera } \\
\text { general que la convivencia de estudiantes } \\
\text { y padres de familia se ve afectada por los } \\
\text { factores del contexto, y que además estas } \\
\text { situaciones presentadas son un punto de } \\
\text { inicio para iniciar un proyecto pertinente que } \\
\text { involucre de manera activa y participativa } \\
\text { a la comunidad educativa y que proporcione } \\
\text { espacios de encuentros para favorecer la sana } \\
\text { convivencia familiar. } \\
\text { En consecuencia, se hace necesario promover } \\
\text { estrategias que generen dinámicas de } \\
\text { transformación en las familias que inviten } \\
\text { a participar de los procesos educativos } \\
\text { escolares, ya que la familia es el núcleo de } \\
\text { la sociedad y a su vez un punto de partida } \\
\text { indispensable en la formación de los } \\
\text { educandos, encargada de trasmitir valores } \\
\text { a los hijos, a fin de que se conviertan en } \\
\text { ciudadanos virtuosos. }\end{array}$ \\
\hline $\begin{array}{l}\text { Huertas } \\
\text { caseras }\end{array}$ & $\begin{array}{l}\text { ¿Sabe usted que } \\
\text { es una huerta } \\
\text { casera?, ¿Qué } \\
\text { tipo de recursos } \\
\text { se necesitan } \\
\text { para la creación } \\
\text { de una huerta } \\
\text { casera familiar? }\end{array}$ & $\begin{array}{l}\text { Los padres y educandos } \\
\text { responden que "conocen } \\
\text { las huertas caseras ya que } \\
\text { el contexto en el cual está } \\
\text { inmersa esta comunidad se } \\
\text { dedican en su mayoría a las } \\
\text { labores de campo. Aunque } \\
\text { los entrevistados no han } \\
\text { tenido la oportunidad de } \\
\text { tener una huerta en casa; } \\
\text { Sin embargo, al responder } \\
\text { la pregunta número tres } \\
\text { estos expresaron que los } \\
\text { recursos que se necesitan } \\
\text { para ello son: abonos } \\
\text { naturales, campos, siscos, } \\
\text { arena de arroyos, así como } \\
\text { escoger las semillas de } \\
\text { los frutos que se quieran } \\
\text { cultivar demostrando que } \\
\text { tienen claridad acerca de los } \\
\text { recursos que se necesitan } \\
\text { para la misma. }\end{array}$ & $\begin{array}{l}\text { De acuerdo a las respuestas se deduce } \\
\text { que los estudiantes y padres de familia en } \\
\text { algún momento de su vida han sembrado } \\
\text { hortalizas o árboles, en sus casas en la calle, } \\
\text { en el colegio, y que para llevarlo a cabo } \\
\text { lo primero que hacen es buscar un lugar } \\
\text { adecuado donde plantarlos es decir que se } \\
\text { encuentre en óptimas condiciones para el } \\
\text { cultivo. Asimismo, el Huerto casero familiar } \\
\text { se compone de diferentes áreas de manejo, } \\
\text { caracterizadas por el uso que se les da. Estas } \\
\text { incluyen diversas combinaciones de especies } \\
\text { animales y vegetales y variedades de árboles, } \\
\text { arbustos y plantas Soto, (2011). } \\
\text { Cabe resaltar que El huerto familiar tiene } \\
\text { especial importancia porque contribuye a } \\
\text { asegurar la alimentación y nutrición de la } \\
\text { familia participando estas en las actividades } \\
\text { productivas. La existencia en él de plantas } \\
\text { medicinales, aromáticas y frutales permite } \\
\text { conservar la tradición de la medicina natural. } \\
\text { Con los productos del huerto además de } \\
\text { abastecerse la familia, lo que representa } \\
\text { un ahorro importante se puede mejorar los } \\
\text { ingresos vendiendo los excedentes (Arias } \\
2012 ; \text { Rivas y Rodríguez } 2013 \text { ) referido en } \\
\text { Universidad de Costa Rica (2014). } \\
\text { Las huertas caseras generan espacios de } \\
\text { participación e integralidad en las familias, } \\
\text { brindan ingresos y permiten el buen uso del } \\
\text { tiempo libre en actividades productivas. }\end{array}$ \\
\hline
\end{tabular}




\begin{tabular}{|c|c|c|c|}
\hline $\begin{array}{c}\text { Categoría de } \\
\text { estudio }\end{array}$ & $\begin{array}{c}\text { Pregunta } \\
\text { orientadora }\end{array}$ & Discurso de los actores & Articulación y sistematización teórica \\
\hline $\begin{array}{l}\text { Relaciones } \\
\text { interpersonales }\end{array}$ & $\begin{array}{l}\text { ¿Considera que } \\
\text { el desarrollo } \\
\text { de las huertas } \\
\text { caseras es } \\
\text { una buena } \\
\text { herramienta } \\
\text { para fortalecer } \\
\text { y mejorar las } \\
\text { relaciones } \\
\text { interpersonales } \\
\text { de la comunidad } \\
\text { educativa de } \\
\text { El Retiro?; } \\
\text { ¿Además de } \\
\text { mejorar la } \\
\text { convivencia } \\
\text { casera, } \\
\text { que otros } \\
\text { benéficos puede } \\
\text { generar la } \\
\text { implementación } \\
\text { de una huerta } \\
\text { casera? }\end{array}$ & $\begin{array}{l}\text { Sí es una herramienta que } \\
\text { permite fortalecer la sana } \\
\text { convivencia, promueve el } \\
\text { trabajo en equipo, estimula } \\
\text { la confianza, el dialogo, } \\
\text { autoestima, y mejora las } \\
\text { relaciones interpersonales, } \\
\text { además favorece el } \\
\text { compartir y la colaboración } \\
\text { de actividades en común } \\
\text { acuerdo. } \\
\text { Es una labor divertida } \\
\text { y social, que fomenta la } \\
\text { investigación, el buen } \\
\text { uso de las TIC como una } \\
\text { herramienta de apoyo para } \\
\text { estos procesos. }\end{array}$ & $\begin{array}{l}\text { Los educandos a través de la implementación } \\
\text { de las huertas caseras, participaran de } \\
\text { manera activa en la ejecución del proyecto, } \\
\text { logrando así adquirir competencias que } \\
\text { les permitan ser capaces de transformar } \\
\text { su realidad y de buscar solución a las } \\
\text { problemáticas que se presenten en su entorno. } \\
\text { Según estudio realizado por la Universidad } \\
\text { De Pamplona en el año } 2012 \text {, las huertas } \\
\text { caseras desde hace mucho tiempo son de } \\
\text { gran beneficio para suplir necesidades tanto } \\
\text { alimentarias como para la venta y recaudo } \\
\text { para beneficio de la escuela, pues es de } \\
\text { vital importancia crear en el estudiante un } \\
\text { espíritu de emprendimiento y fortalecimiento } \\
\text { por la producción de huertas productivas para } \\
\text { beneficio propio. }\end{array}$ \\
\hline
\end{tabular}

Fuente: elaboración propia. 2018.

\section{Conclusiones}

De acuerdo a las consideraciones más relevantes del proyecto, se destacan los siguientes aportes:

- Promoción del respeto y el principio de la conservación del medio ambiente.

- Vinculación de los padres, estudiantes, profesores, en el desarrollo de huertas caseras, para la obtención de alimentos sanos y nutritivos que permiten una salud adecuada en los hogares

- La articulación de los proyectos en diferentes programas pedagógicos que se aplican en las distintas áreas del conocimiento.

- Aplicar las huertas caseras permite mejorar las relaciones interpersonales y la convivencia en la comunidad educativa
- Permite la integración de familias expertas en el manejo, cuidado del cultivo y cosecha de las huertas.

Cabe destacar la importancia y el liderazgo por parte de los estudiantes, padres y docentes en cuanto al acompañamiento, la pertinencia, la responsabilidad y el seguimiento durante el desarrollo de este proyecto que promociona el logro de aprendizajes significativos para obtener resultados esperados en los procesos de enseñanza aprendizajes.

\section{Referencias}

Arias. M. (2013). Huertos caseros en Costa Rica y América. Ambientico, 3 (243). 2-31. Recuperado de http://www.ambientico.una.ac.cr/pdfs/ambientico/243. pdf. 
Blaya, C., Debarbieux, E., Alamillo, R. y Ortega, R. (2006). Clima y violencia escolar. Un estudio comparativo entre España y Francia. Revista de Educación, 339, 293-315.

Cruz, E. (2016). El ciclo de protesta 2010 2016 en Colombia. Una explicación. Jurídicas CUC, 12 (1), 31-62. http://dx.doi. org/10.17981/juridcuc.12.1.2016.3.

Erazo, O. (2010). Reflexiones sobre la violencia escolar. Revista de Psicología GEPU, 1(3), 74 - 86.

Forero, O. (2011). La violencia escolar como régimen de visibilidad. Magis, Revista Internacional de Investigación en Educación, 4(8).

Gómez, H. (2015). Evaluación de la estrategia pedagógica de huertas caseras para mejorar la nutrición y desempeño escolar en la Institución Educativa Agropecuaria "Santa Rita", La Vega Cauca. [Tesis de Pregrado]. Universidad Nacional Abierta y a Distancia UNAD. Popayán, Colombia.

Gómez, L. y Ortega, W. (2012). La huerta Escolar y las TIC para un mejor futuro. Campamento, Antioquia. Recuperado de http://wadithortega.wordpress. com/2012/11/25/la-huerta-escolar-y-lastic-para-un-mejor/

Guerrero, H. y Cepeda. (2016) El impacto del lider comunitario en el siglo XXI. Barranquilla: Editorial Universitaria De La Costa.

Herrera-Tapia, B. (2014). Las acciones colectivas en Colombia frente a una realidad global: El derecho de consumo. Justicia, 25(1). 70-81.

Instituto Agustin Codazzi. (2015). Instituto-Geográfico-Agustín-Codazzi. Recuperadodehttp://studylib.es/doc/7199891/ ley-1753-de-2015 - - - instituto geogr\%C3\%A1fico-agust\%C3\%ADncodazzi
Jares, X. (2008). Formación del Profesorado para la convivencia. En, MEC, IV Congreso de Convivencia, Profesorado y Convivencia. Madrid, España. Recuperado de http://multiblog.educacion. navarra.es/iibarrog/files/2010/04/Jares.pdf

Mazzini. C. (2012). Secuencias didácticas $y$ huerta escolar. Recuperado de http:// arconrecursosisfd119pep.blogspot.com. co/p/blog-page.html.

Palacios, J., Amud, N. y Perez, D. (2016). implementación de huertas escolares como estrategia de enseñanza-aprendizaje de la biología de grado sexto en la Institución Educativa Agrícola de Urabá del Municipio de Chigorodó y de grado séptimo de la Institución Educativa Rural Zapata, De Necoclí, Departamento de Antioquia. [Tesis de Maestría]. UniversidadPontificaBolivariana. Medellín, Colombia.

Palacios, M. (2016). Implementación de huertas escolares como estrategia de enseñanza aprendizaje. Medellín: catarina.udlap.mx. (s.f.). Recuperado de http://catarina.udlap.mx/u_dl_a/tales/ documentos/lco/oropeza_c_f/capitulo4. pdf

República de Colombia. Constitución Política de Colombia. (1991). www.constitucioncolombia.com. Recuperado de http://www.constitucioncolombia.com/ titulo-2/capitulo-2/articulo-44

Rivas, P. y Rodríguez. L (2013). Huertos caseros en Costa Rica y América. Ambientico, 3(243). 2-31.Recuperado de: http://www.ambientico.una.ac.cr/pdfs/ ambientico/243.pdf.

SEE. (2009). El Huerto escolar como recurso de enseñanza-aprendizaje de las asignaturas del curriculo de educación básica. Recuperado de http://www.fao. org/ag/humannutrition/21877061e61334701c700e0f53684791ad06ed.pdf 
Soto, A. (2011). Las áreas verdes urbanas: Una alternativa para mejorar el microclima urbano. Cultura y Sociedad, 1(1).
Vera, J. (2015). La huerta escolar como estrategia didáctica para el desarrollo de competencias científicas en la Institución Educativa Maestro Pedro Nel Gómez. [Tesis de Maestría]. Universidad Nacional de Colombia. Sede Medellín. 